Article

\title{
A Classical Based Scalar Field Model Provides a Fundamental Bridge Between General Relativity Effects and Quantum Mechanics
}

Rickey Austin 1,† 0000-0002-5833-9272

1 St Claire Scientific Research, Development and Publications; rickeywaustin@stclairesrdp.com

$\dagger$ Current address: Albuquerque, NM

\begin{abstract}
Herein is introduced a simple scalar field model derived from classical based kinetic energy, gravitational potential energy, and Special Relativity's rest mass energy. By applying a classical orbit over the scalar field, relativistic effects are predicted. The scalar field is then applied to a classical model of the Hydrogen atom resulting in a relativistic effect equal to the binding energy of the Hydrogen atom. In addition, the model derives the fine structure constant due to the gravitational effect. The relativistic effects are then discretized in increments equal to the model's gravitational induced constant. The discretization produces the Hydrogen atom spectral emissions and an angular moment equal to Planck's reduced constant. The model is not presented as a replacement for current theory, rather it is for inspection and illustration of how a simplistic model may offer a fundamental bridge between the more complex, time proven theories of General Relativity and Quantum Mechanics.
\end{abstract}

Keywords: Classical Model; General Relativity; Hydrogen Atom, Fine Structure Constant, Planck Constant, Dark Energy, Dark Matter

\section{Introduction}

As part of my doctoral thesis a method was derived for introductory teaching of General Relativity (GR) via starting with Newtonian laws [1]. From Newtonian laws GR effects are derived to first order equality in Schwarzschild's solution to Einstein's field equations. The derivation methodology follows the same method as when Special Relativity is derived by limiting the speed of particles to the speed of light in a vacuum [2]. In a continuation of the study I introduce here a scalar field $S$ derived from Newtonian Gravitational potential and classical kinetic energy. By applying Newtonian mechanics over $S$, it is shown GR effects are included in the Newtonian calculations. This method when applied to celestial orbits predicts a perihelion advance. The results of the calculations provide high fidelity compared to known measurements and mainstream theories.

Next, the field $S$ is applied to a classical notion of an electron orbiting a proton. In this application of $S$, an electron orbital advance is predicted. The model shows in addition to the classical Coulomb binding energy, an additional binding energy resulting from the orbital advance. The total binding energy is evaluated when an interacting photon has a wavelength equal to the reduced Compton wavelength for the electron. At this equality, the additional binding energy due to the advance is equal the binding energy of Bohr's Hydrogen model for the atom [? ], $27.2 \mathrm{eV}$.

\section{Introduction}

Here a scalar field model $S$ is generated by equating a virtual point mass's classical kinetic and potential energy as a ratio to the rest energy of the mass generating the field. Then the model is used to investigate time dilation as an effect of the field due to the mass. The results are then compared to Schwarzschild's solution[3] to Einstein's field equations for matching accepted theory. Next, the field $S$ is applied to a classical based model of an electron orbiting a proton. In this investigation of $S$, an electron orbital advance is predicted. The model shows in addition to the classical Coulomb binding energy[4], an additional binding energy resulting 
from the orbital advance. The model's total binding energy is evaluated when a photon with wavelength equal to the reduced Compton wavelength[5] interacts with the electron. At this equality, the additional binding energy due to the advance is equal the binding energy of the Hydrogen atom $(13.6 \mathrm{eV})[5]$.

\section{The Scalar Field Model}

The scalar field is derived from Newtonian gravitational potential and classical kinetic energy[4]. The gravitational potential is mapped to the field by considering a virtual point particle $\left(m_{v}\right)$ equal to the mass $(M)$ generating the field. The gravitational potential of $m_{v}$ is calculated for all space with radial distance greater than or equal to the radius of $M$. The potential value at each point is equated by:

$$
V_{g}=-\frac{G M m_{v}}{r^{2}}
$$

Where $G$ is the Newtonian Gravitational constant[5] and $r$ is the radial distance from the center of mass $M$ to the point mass $m_{v}$. Secondly the kinetic energy of $m_{v}$ relative to $M$ is calculated by:

$$
T_{g}=\frac{m_{v} v^{2}}{2}
$$

Where $v$ is the instantaneous velocity of $m_{v}$ relative to $M$. The energies in equations ( $1 \& 2$ ) when combined form the classical total energy of $m_{v}$ [6]. Lastly, the total energy is evaluated as a ratio to $M$ 's rest mass's energy and added to unity. The proportionality is a dimensionless scalar value:

$$
A=1+\frac{T_{g}-V_{g}}{E_{0}}
$$

It is here hypothesized the value $A$ represents the scalar time rate of a location in the field, proportional to the time rate at a location where no gravitational influence exist. To transform the field's observed time rate at a location $\left(t_{\text {local }}\right)$ to the observed time rate at infinity $\left(t_{\infty}\right)$, apply equation 3 thus forth:

$$
t_{\infty}=A \cdot t_{\text {local }}
$$

Here the term Exemplar space is introduced for convenience, to represent any space where there is no gravitational influence. In Exemplar space, the value of A by definition is 1 .

$$
A_{\text {Exemplar }} \equiv 1
$$

In following Einstein's postulate of the universal observed speed of light (c) [7], locally observed measurements of time and length are determined by the distance a ray of light travels in one unit of time $(\hat{t})$ as measured locally. The unit of length $(\hat{l})$ is:

$$
\hat{\imath}=c \hat{t}
$$

From any location within the field, the unit length and unit time are related to the unit length and unit time in Exemplar space by:

$$
\hat{l}_{\text {exemplar }}=A_{\text {local }} l_{\text {local }}
$$

Where $A$ is locally calculated. From this relatively non-complex scalar field, utilizing transformations from local space to Exemplar space, General Relativity effects are investigated. 


\section{Relativistic effects in $S$}

\subsection{Time Dilation}

Using equation 3 , a clock $c_{1}$ at radial distance $r_{1}$ is compared to a clock $c_{E 1}$ in Exemplar space:

$$
c_{E 1}=A_{1} c_{1}=\left(1+\frac{v_{1}^{2}}{2 c^{2}}+\frac{G M}{r_{1} c^{2}}\right) c_{1}
$$

Where $A_{1}$ is the calculated field value at location $r_{1}$ from the center of mass $M$. When $v_{1}$ equals zero:

$$
c_{E 1}=A_{1} c_{1}=\left(1+\frac{G M}{r_{1} c^{2}}\right) c_{1}
$$

In Schwarzschild's metric [3], at a radial distance $r$ from the center of mass $M$ with zero radial velocity, the local clock rate $\tau$ as compared to a clock rate $(t)$ an infinite distance from the mass $(M)$ is:

$$
t=\frac{1}{\sqrt{1-\frac{G M}{r c^{2}}}} \tau
$$

Performing a binomial expansion on equation 10 for $\frac{G M}{r c^{2}}$ :

$$
t=\left(1+\frac{G M}{r c^{2}}+\ldots\right) \tau
$$

Analyzing equations 9 and 11 the model produces an equivalent measurement of time dilation to a minimum first order equivalence of Schwarzschild's metric in GR.

\subsection{Orbits in the Scalar Field Model}

Consider a circular orbit in $S$ about a massive object. To calculate the orbital transformation from a non-exemplar space to exemplar space requires squaring the value of $A$. This is because both distance and time are transformed. The transformation of the circumference $C_{l}$ to $C_{E}$ takes the form:

$$
C_{E}=2 \pi A r=2 \pi\left(1+\frac{G M}{2 r c^{2}}+\frac{G M}{r c^{2}}\right)^{2} r
$$

Where the potential is calculated from a gravitational orbit. Of interest, equation 12 shows for any gravitational circular orbit there will be a first order advance of:

$$
\text { advance }=2 \pi\left(1+\frac{3 G M}{2 c^{2}}\right)^{2}
$$

Note the radial value $r$ cancels out and one is left with a constant advance in length for all circular orbits regardless of radial distance from the central massive object. For orbits that are not circular but elliptical, the radial distance can be calculated as a dependency on the angle of rotation[8] by setting $r$ equal to:

$$
r=\frac{a\left(1-e^{2}\right)}{1+e \cos (\theta)}
$$

Where $a$ is the semi-major axis, e is the eccentricity, and $\theta$ is the angle of rotation. Integrating the value for $\theta$ over the angle of $2 \pi$ for one period $P$ yields the advance. The full calculation to first order of the model is:

$$
\int_{0}^{1} \int_{0}^{2 \pi}\left(\left(1+\left(\frac{3 G M}{2\left(\frac{a\left(1-e^{2}\right)}{1+e \cos (\theta)}\right) c^{2}}\right)\right)^{2}-1\right) d \theta d P
$$

The -1 is applied such that only the advance is calculated and not the entire elliptical circumference. When equation 15 is applied to the planet Mercury, it predicts an advance of 42.98 arc seconds per century which agrees closely to observations [9] and General Relativity effects. 


\section{Classical Model of the Hydrogen Atom in $S$}

In the classical approach, the electron is modeled to orbit the proton in the same way a planet orbits the sun. In modeling the Hydrogen atom the classical Coulomb equation is implemented into $S$ for equating A:

$$
A(r, m, v)=1+\frac{e^{2}}{8 \pi \epsilon_{0} m_{e} r c^{2}}+\frac{G M_{p}}{2 r c^{2}}+\frac{G M_{p}}{r c^{2}}
$$

Where $M_{p}$ is the proton mass, $\epsilon_{0}$ is vacuum permittivity, $r$ the radial distance from the center of the proton, $e$ is the elementary charge, and $c$ is the speed of light. Equation 16 is squared in the same manner as with the celestial orbit:

$$
A(r, m, v)^{2}=1+\frac{e^{2}}{4 \pi \epsilon_{0} m_{e} r c^{2}}
$$

The second order contributions and the gravitational potential have been omitted in equation 17 as their contributions are very small compared to the Coulomb contribution. As with the gravitational orbit, when calculating an advance in orbit, the additional distance traversed per revolution in a circular orbit is:

$$
\text { Advance }=2 \pi\left(\frac{e^{2}}{4 \pi \epsilon_{0} m_{e} c^{2}}\right)
$$

Equation 18 is for a circular orbit. It is assumed any observed measurement will predict an orbital period as starting and ending at the same point for each completed orbit (in a circular orbit). This orbital observance omits any advance in the orbital system. As such, according the the model, the total binding force of the orbit will be greater than the classically predicted binding energy.

\subsection{Electron Binding Energy of the Hydrogen Atom in $S$}

In analyzing the model's predicted additional binding energy due to the orbital advance, the classical Coulomb potential energy[10] is considered:

$$
E_{C}=\frac{e^{2}}{4 \pi \epsilon r}
$$

along with the additional energy needed to traverse the advance in equation 18 . If one takes the ratio of the advance to the circumference of the orbit, it is:

$$
\text { ratio }=\frac{a d v}{2 \pi r}=\frac{\left(\frac{a d v}{2 \pi}\right)}{r}
$$

Using the value from equation 20 and multiplying it by the Coulomb energy, the additional energy is derived. The additional energy due to the advance is:

$$
E_{A}(r)=\left(\frac{e^{2}}{4 \pi \epsilon r}\right)\left(\frac{\left(\frac{a d v}{2 \pi}\right)}{r}\right)
$$

The derived total energy $E_{T}(r)$ is obtained by equating the energy of the classical orbit $E_{C}(r)$ plus the additional energy due to the orbital advance $E_{A}(r)$ as a function of radial distance $r$ :

$$
E_{T}(r)=E_{C}(r)+E_{A}(r)=\frac{e^{2}}{4 \pi \epsilon r}+\left(\frac{e^{2}}{4 \pi \epsilon r}\right)\left(\frac{\left(\frac{a d v}{2 \pi}\right)}{r}\right)
$$

where $r$ is the radial distance between the proton and electron. 


\subsection{Photon Interaction with the Hydrogen Atom in $S$}

The energy of a photon with wavelength $r$ is[10]:

$$
E_{\gamma}(r)=\frac{h c}{r}
$$

Setting equation 23 equal to $E_{T}(r)$ (equation 22) and adding variable $K$ as the value of proportionality between $E_{\gamma}(r)$ and $E_{T}(r)$ :

$$
K\left(\frac{h c}{r}\right)=\frac{e^{2}}{4 \pi \epsilon r}+\left(\frac{e^{2}}{4 \pi \epsilon r}\right)\left(\frac{\left(\frac{a d v}{2 \pi}\right)}{r}\right)
$$

Solving for $K$ :

$$
k=\left(\frac{\left(\frac{e^{2}}{4 \pi \epsilon r}\right)}{\left(\frac{h c}{r}\right)}\right)+\left(\frac{\left(\left(\frac{e^{2}}{4 \pi \epsilon r}\right)\left(\frac{\left(\frac{a d v}{2 \pi}\right)}{r}\right)\right)}{\left(\frac{h c}{r}\right)}\right)
$$

Reducing and rearranging equation 25 and using equation 18 for the advance value:

$$
K=\frac{1}{2 \pi}\left(\frac{e^{2}}{2 \epsilon_{0} h c}+\frac{e^{2}}{2 \epsilon_{0} h c}\left(\frac{a d v}{2 \pi r}\right)\right)
$$

From equation 24, the photon energy equivalent to $E_{T}(r)$ as a function of $\lambda$ is:

$$
E_{\gamma}(\lambda)=\overbrace{\underbrace{\frac{E_{C}(\lambda)}{K(\lambda)}}_{\text {Coulomb } / \mathrm{K}}+\underbrace{\frac{E_{A}(\lambda)}{K(\lambda)}}_{\text {Advance } / \mathrm{k}}}^{\text {Total Potential Energy }}
$$

When one investigates $\lambda=\lambda_{e}$ (the reduced Compton wavelength[5] ) equation 27 generates the following values:

$$
\begin{aligned}
\lambda & =\lambda_{e} \\
\text { Advance } & =2 \pi r_{e} \\
\text { Ratio } & =\alpha \\
r=\lambda_{e} & =\frac{r_{e}}{\alpha} \\
E_{\gamma}\left(\lambda_{\tau}\right) & =2 \pi m_{e} c^{2} \\
E_{C}(r) & =\alpha m_{e} c^{2} \\
E_{A}(r) & =\alpha^{2} m_{e} c^{2}
\end{aligned}
$$

Where $\alpha$ is the fine structure constant[5], $r_{e}$ is the classical electron radius[5], and $m_{e}$ is the mass of the electron. For a visual representation of the list of equations and values see Figure 1. The equations are derived from the predicted advance of the electron orbit. The advance causes an additional energy $E_{A}(r)$ equated as traditional Coulomb energy $E_{C}^{\prime}\left(r^{\prime}\right)$. The potential/binding energies allowable and the angular momentum states allowable energy occur at discrete energy levels.

If an investigator examined the energy from $E_{A}(r)$ it is logical to imagine they would consider it a Coulomb based potential energy. As such, from a classical viewpoint the energy 


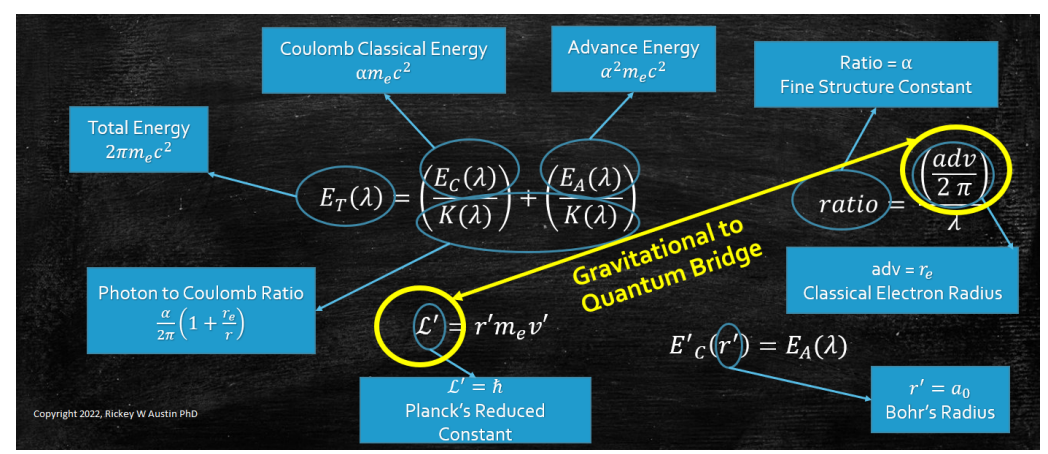

Figure 1. Equation Breakout

would relate to equation 19. In terms of classical Coulomb energy, the advance induced energy is:

$$
E_{C}^{\prime}\left(r^{\prime}\right)=\frac{e^{2}}{4 \pi \epsilon r^{\prime}}=E_{A}(r)=\left(\frac{e^{2}}{4 \pi \epsilon r}\right)\left(\frac{\left(\frac{a d v}{2 \pi}\right)}{r}\right)
$$

To remove ambiguity the new Coulomb based equation representing an investigator's evaluation of $E_{A}(r)$ is marked with a prime symbol. The value of $E_{A}(r)$ when $\lambda=\lambda_{e}$ is:

$$
E_{A}(r)=\alpha^{2} m_{e} c^{2}=E_{C}^{\prime}\left(r^{\prime}\right)
$$

The energy in equation 37 is equivalent to the classical potential energy of the electron to the proton in the Hydrogen atom $(27.12 \mathrm{eV})$.

Recall from the model, the advance of the electron (equation 29) is an invariant distance $2 \pi r_{e}$ for any orbit. If one increments the radial magnitude by integer multiples of $r_{e}$, discrete energy levels are generated. Using equation 36 and solving for $r^{\prime}\left(\right.$ when $\lambda=\lambda_{e}$ ):

$$
r^{\prime}=\frac{\left(\frac{r_{e}}{\alpha}\right)^{2}}{r_{e}}
$$

Discretize equation 38 in increments of $r_{e}$ by adding the value $n$ to the equation where $n=$ $\{1,2,3, . . n\}$ :

$$
r^{\prime}(n)=\frac{\left(\frac{n r_{e}}{\alpha}\right)^{2}}{r_{e}}
$$

One can now ascertain the conversion of $E_{C}^{\prime}\left(r^{\prime}\right)$ into a step function of $r_{e}$ (the gravitational induced constant of the electron orbital advance) by:

$$
\frac{e^{2}}{4 \pi \epsilon r^{\prime}}=\frac{e^{2}}{4 \pi \epsilon r} \rightarrow r^{\prime}(n)=\frac{\left(\frac{n r_{e}}{\alpha}\right)^{2}}{r_{e}}=\frac{n^{2} r_{e}}{\alpha^{2}}
$$

Thus the energy in equation 36 has discrete allowable energy magnitudes via the following equation:

$$
E_{C}^{\prime}(n)=\frac{e^{2}}{4 \pi \epsilon \frac{n^{2} r_{e}}{\alpha^{2}}}=\frac{\alpha^{2} e^{2}}{4 \pi \epsilon_{0} n^{2} r_{e}}
$$


Interpreting equation 41 the total potential energy of the Hydrogen atom may only take on discrete values due to the advance of the electron (a gravitational effect). Thus, the following potential energies are allowed for the system:

$$
\begin{aligned}
E_{C}^{\prime}(1) & =\frac{\alpha^{2} e^{2}}{4 \pi \epsilon_{0}\left(1^{2}\right) r_{e}} \\
E_{C}^{\prime}(2) & =\frac{\alpha^{2} e^{2}}{4 \pi \epsilon_{0}\left(2^{2}\right) r_{e}} \\
\ldots & \\
E_{C}^{\prime}(n) & =\frac{\alpha^{2} e^{2}}{4 \pi \epsilon_{0} n^{2} r_{e}}
\end{aligned}
$$

According the Virial theorem [11] the average kinetic energy of an orbiting particle will have half the average potential energy of the bound system [12]. Thus, to remove an electron from an $n$ state energy, $\frac{1}{2} E_{C}^{\prime}(n)$ is required. Listed below are the values for the first three states.

$$
\begin{aligned}
& \left(\frac{1}{2}\right) E_{C}^{\prime}(1)=13.6 \mathrm{eV} \\
& \left(\frac{1}{2}\right) E_{C}^{\prime}(2)=3.40 \mathrm{eV} \\
& \left(\frac{1}{2}\right) E_{C}^{\prime}(3)=1.51 \mathrm{eV}
\end{aligned}
$$

If one considers the difference between energy levels, the differential energies match the Hydrogen spectral emissions [13]:

$$
\begin{aligned}
& \left(\frac{1}{2}\right) E_{C}^{\prime}(1)-\left(\frac{1}{2}\right) E_{C}^{\prime}(2)=10.20 \mathrm{eV} \\
& \left(\frac{1}{2}\right) E_{C}^{\prime}(1)-\left(\frac{1}{2}\right) E_{C}^{\prime}(3)=12.09 \mathrm{eV}
\end{aligned}
$$

These are representative of the Lyman series [14]. Other series may be derived in a similar manner.

\subsection{Hydrogen Atom's Electron Angular Momentum in the Scalar Field Model}

A natural continuation of analyzing the model is to classically calculate the angular momentum of an electron in orbit around a proton. In order to calculate angular momentum, velocity is required. From deriving equation 16 the velocity as a function of $r$ is:

$$
v(r)=\sqrt{\frac{e^{2}}{4 \pi \epsilon_{0} m_{e} r}}
$$

Using the value of $r=\frac{r_{e}}{\alpha}$ from equation 29, $v(r)$ is:

$$
v(r)=\sqrt{\frac{\alpha e^{2}}{4 \pi \epsilon_{0} m_{e} r}}
$$

Combining the radius, mass, and velocity the classically derived angular momentum at $r$ is:

$$
\mathscr{L}_{T}(r)=r m v=\sqrt{\frac{r_{e} m_{e} e^{2}}{4 \pi \epsilon_{0} \alpha}}
$$


If one steps the radial value by integer multiples of $r_{e}$ equation 55 is transformed to a discrete equation:

$$
\mathscr{L}_{T}(n)=r m v=\sqrt{\frac{n r_{e} m_{e} e^{2}}{4 \pi \epsilon_{0} \alpha}}
$$

Where $n$ takes on an integer value $\{1,2,3 .$.$\} . Next, equate the angular momentum \mathscr{L}^{\prime}$ using equation 38 for $r^{\prime}$ :

$$
\mathscr{L}^{\prime}(n)=\left(\frac{\left(\frac{n r_{e}}{\alpha}\right)^{2}}{r_{e}}\right) m_{e} \sqrt{\left(\frac{e^{2}}{4 \pi \epsilon m_{e}\left(\frac{\left(\frac{n r_{e}}{\alpha}\right)^{2}}{r_{e}}\right)}\right)}=n \hbar
$$

\section{Discussion and Conjectures}

Equation 57 provides a straight forward understanding of the model's prediction. The angular momentum of the system must step in discrete increments of energy. When $\lambda=\lambda_{e}$ the discrete energy step is $h$. This correlation shows a one to one relation between the gravitational induced advance of the electron and the discrete angular momentum levels allowed. This provides a direct link from gravitational effects to a causal effect of quantum discrete energy levels in the angular momentum of an electron proton system. The model predicts a photon of wavelength $\lambda_{e}$ interacting with the orbital will only generate a portion of the total energy (13.6 $\mathrm{eV}$ ). This implies there may be a violation of conservation of energy within the model.

\subsection{Contracting Space-Time}

As a possible explanation for why only the $E_{A}$ portion of energy is emitted with an incoming photon, it is here conjectured that a photon's interaction with the system only creates or absorbs photons when space-time is contracting or expanding. This conjecture leads to the following postulate for the model: Photon creation and absorption only happens when there is a change in the curvature of space-time. Mathematically:

$$
\Delta C= \begin{cases}\text { absorption, } & \text { When } \Delta \mathrm{C} \text { is positive } \\ \text { creation, } & \text { When } \Delta \mathrm{C} \text { is negative }\end{cases}
$$

Where $C$ is the curvature of space-time. When the change in curvature is positive (increasing - away from zero curvature) there is an energy increase in the system causing the change in space-time curvature, thus the energy was adsorbed. When the change in curvature is negative (decreasing - toward zero curvature) there is an energy decrease in the system causing the change in space-time curvature, thus energy is created (photon). When over a period of time $\Delta C=0$ there will be no absorption or creation of photons. For clarity, the postulate states when a change of the curvature of space-time happens (an event), the event generates either an absorption or creation of a photon. If there is no event (i.e. change in the curvature of space-time) then no photon is absorbed or created. The generated photon is proportional to the change in the curvature of space-time.

How does this relate to the conservation of energy? If the postulate holds, then the incoming energy of the photon would have been considered only $13.6 \mathrm{eV}$, and not $2 \pi m_{e}$. This is because the previous interaction with matter to measure the interaction of the photon would have been measured by the event of the change in the curvature of space (thus only considered $13.6 \mathrm{eV}$ ). In other words, the additional energy would be hidden from observation because its interaction did not create or absorb a photon equal to the total energy, but to the observed energy as generated by the change in curvature of space-time.

\section{Conclusion}

In conclusion it has been shown a scalar field model produces results comparable to Schwarzschild's solution to Einstein's field equations. In applying the model to a classical model 
of the Hydrogen atom, an unexpected prediction arose. The model predicted an additional binding energy to the classical model that is equivalent to the binding energy of the Hydrogen atom when the energy of the system is equated to the rest mass energy of the electron. The derived gravitational induced constant angle of advance of the electron's orbit is the fine structure constant. In addition, when discretized, the model provides accurate spectral emissions for the Hydrogen atom and a one to one relation between Planck's reduced constant $h$ and the orbital advance caused by the gravitational effect.

Understanding the reason(s) the model presents accuracy to empirical data when the system's energy is equivalent to the rest energy of the electron merits further research. Although by adding a strong and far reaching postulate, an explanation may exist. The postulate would help explain why only the gravitational predicted effect generates photons, and only generates them when space-time contracts. This is a postulate of great impact across many disciplines and needs much research, vetting, before fully implementing it into the field model.

Another area of investigation is the amount of energy the model predicts that is not representative of a photon-matter interaction. Recall the model only shows the gravitational effect generating a photon. As such there is a predicted ratio of $\alpha^{-2}$ of photon observed energy to non-luminous energy. If such an energy exits it may prove beneficial in the hunt for dark matter or dark energy. These conjectures coupled with the model's initial agreement with empirical data and accepted theory warrants deeper investigation, as well as if there are any physical relevancy to the model's predictions.

Funding: The Author wishes to thank Saint Claire Scientific Research and Publications for providing funding making this research possible. 


\section{References}

1. Austin, R.W. A Classical Based Derivation of Time Dilation Providing First Order Accuracy to Schwarzschild's Solution of Einstein's Field Equations. PhD thesis, North Carolina Agricultural and Technical State University, 2017.

2. Austin, R.W. Gravitational Time Dilation Derived from Special Relativity and Newtonian Gravitational Potential. European Scientific Journal, ESJ 2017, 13, 447. doi:10.19044/esj.2017.v13n3p447.

3. Schwarzschild, K. On the gravitational field of a mass point according to Einstein's theory, 1999, [arXiv:physics.hist-ph/physics/9905030].

4. Giancoli, D.C. Physics for Scientists and Engineers, second ed.; Prentice Hall, 1989.

5. CODATA. 2018 .

6. Marion, J.B. Classical Dynamics of Particles and Systems, second ed.; Academic Press, 1970.

7. Einstein, A. The Formal Foundation of the General Theory of Relativity. NEED 1914.

8. Misner, Thorne, W. Gravitation, first ed.; W H Freeman and Company, 1970.

9. $\quad$ Park, R.S.; Folkner, W.M.; Konopliv, A.S.; Williams, J.G.; Smith, D.E.; Zuber, M.T. Precession of Mercury's Perihelion from Ranging to theMESSENGERSpacecraft. The Astronomical Journal 2017, 153, 121. doi:10.3847/1538-3881/aa5be2.

10. Giancoli, D.C. Physics for Scientists and Engineers, second ed.; Vol. 2, Prentice Hall, 1989.

11. Stephen T. Thornton, A.R. Modern Physics For Scientists and Engineers, 2 ed.; Brooks/Cole, 2002.

12. Carroll, B.; Ostlie, D. An Introduction to Modern Astrophysics, second ed.; Pearson - Addison Wesley, 2007.

13. Kramida, A., Ralchenko, Yu., Reader, J. and NIST ASD Team (2021). NIST Atomic Spectra Database (version 5.9), 2022. doi: $\{10.18434 / \mathrm{T} 4 \mathrm{~W} 30 \mathrm{~F}\}$.

14. Lyman series; Oxford Reference, 2022. doi:10.1093/oi/authority.20110803100120100. 